\title{
Preprints in biomedicine: alternative or complement to the traditional model of publication?
}

\author{
Guillermo Aquino-Jarquin ${ }^{1}$, Josefina de Monserrat Valencia-Reyes ${ }^{1}$, Abraham Silva-Carmona ${ }^{1,2}$ and
} Javier Tadeo Granados-Riverón ${ }^{1}$

${ }^{1}$ Laboratory of Research in Genomics, Genetics and Bioinformatics, Hospital Infantil de México Federico Gómez; ${ }^{2}$ Pathology Department, Hospital General de México, Ciudad de México, Mexico

\begin{abstract}
The peer-review system has allowed the quality control of the manuscripts submitted for publication to scientific journals for over three centuries. However, due to its relative slowness and other drawbacks, some researchers, mainly in the areas of Physics and Mathematics, started some decades ago to propagate, by electronic means, manuscripts not yet submitted to a journal for formal publication. The dissemination of this practice led to the establishment of permanent repositories like ArXiv, to which preprints can be sent to be published whitou charge, allowing also the search and download of the works they contain with no payment required from the reader. In biomedical sciences, the adoption of the system has been slower than in the exact sciences and previous attempts like e-biomed, Netprints, and Nature Precedings did not prosper. A new generation of repositories like bioRXiv, inspired by ArXiv, seems to enjoy an increasing acceptance among biomedical researchers. Here, we discuss the potential role of this emerging system to establish discovery priority in biomedicine and to improve manuscripts before they are submitted to scientific journals besides other applications which could be implemented in the extent that the model becomes more popular.
\end{abstract}

KEY WORDS: Preprints. Peer-review. Repositories. Scientific publication. Biomedical publishing.

\section{Introduction}

Since its inception, when naturalist Henry Oldenburg implemented it in Philosophical Transactions of the Royal Society in the second half of the $17^{\text {th }}$ century, the peer-review system has been the most widely used mechanism to promote scientific literature quality in the entire world. Except for a brief period that included the use of fax, until a few decades ago, peer-review involved physical exchange of printed texts and illustrations ${ }^{1}$. By the same token, already at the end of the past century, traditional peer-review model effectiveness and consistency have been questioned, as the usefulness of measures proposed ever since to improve the process have also been².
In a traditional publishing process, the journal's editor that receives a manuscript decides, based on its subject, quality, originality and rigor, in addition to the potential interest of the journal's readers in it, whether it should be rejected or else be sent for peer-review, which usually is carried out by two or three experts on the subject. After its analysis, each reviewer issues a recommendation to the editor on whether the manuscript should be accepted for publication, be rejected or else be considered for new analysis once the authors adequately answer the questionings expressed in the reviewers' reports, which can include the request for changes in the manuscript writing, analysis of some data or, frequently, the performance of new experiments. Eventually, after a variable number of

\author{
Correspondence: \\ Javier Tadeo Granados-Riverón \\ Dr. Márquez, 162 \\ Col. Doctores \\ C.P. 06720, Ciudad de México, México \\ E-mail: JavierTGranados@ himfg.edu.mx
}

Date of reception: 18-07-2016

Date of acceptance: 27-07-2016

DOI://dx.doi.org/10.24875/GMM.M18000116
Gac Med Mex. 2018;154:66-70

Contents available at PubMed www.gacetamedicademexico.com 
peer-review rounds, the manuscript is finally accepted for publication or rejected. This entire process can comprise from a few weeks to more than one year and, in case the manuscript is rejected and submitted to another journal, substantially longer.

Although the use of the internet has greatly accelerated the exchange of information used for peer review, recent evidence reveals that average time required between reception and publication of a manuscript in Nature has increased from 85 to little more than 150 days in the past decade, while for PLoS $O N E$, the same parameter has increased from 37 to 125 days in approximately the same period ${ }^{3}$. At least in part, this can be attributed to the increase in the amount of data that are necessary to achieve for a work to be published. A comparison between articles published in Cell, Nature and The Journal of Cell Biology revealed that the number of panels in figures has increased from 2 to 4 -fold between 1984 and $2014^{4}$.

In the last few decades, particularly in biomedicine, both the explosive increase in the number of scientific journals and the resulting increase in the number of annually-published articles have not been accompanied by a proportional increase in the number of potential reviewers. In addition, performing a quality review of an article requires an important investment of time, which, globally, does not yield a proportional direct benefit to it. In the case of Mexico in particular, acting as a reviewer is not recognized as a criterion that facilitates admission to or permanence in agencies such as the National System of Researchers.

This high-volume and little-incentive panorama has with no doubt contributed to decrease peer-review processes rigor and quality, particularly in intermediate and low impact factor publications. For example, scientific misconduct has been estimated to have increased approximately 10 times between 1975 and $2012^{5}$.

\section{Preprints}

In response to traditional peer-review inherent problems, some investigators, especially in the field of physics, chose to directly distribute their discoveries, observations and models to potential readers in the TeX digital format, prior to or instead of submitting them to a journal for peer-review and publication. These preprints or e-prints were initially distributed via e-mail. Early in the nineties, a repository of preprints was created, which now is known as ArXiv and is maintained by the Cornell University. ArXiv is currently available in the internet and has had a huge success, and currently it contains more than one million preprints in the fields of physics, mathematics, computer science, statistics, finance and quantitative biology, which turns it into the main diffusion platform for those disciplines ${ }^{6}$.

In biomedical sciences, the initial proposal to apply the preprint model was named e-biomed, and it was promoted by the US National Health Institutes in $1999^{7}$. Although the idea did not prosper, it gave origin to PubMed Central, a repository that to this day makes a large number of articles published in the traditional system freely available.

Later, both BMJ (Netprints) ${ }^{8}$ and Nature (Nature Proceedings $)^{9}$ started their own preprint servers with biomedical emphasis. Now inactive, both received thousands of works for several years at the beginning of the century, which until today are still available for download. In 2012, F1000research was launched, which is a publishing platform with the open peer-review concept, where the identity of reviewers, as well as the content of all texts originated during the process are published and are downloadable from the platform together with the original version and all subsequent versions of the work. Although F1000research does apply typographic composition and editing to the manuscripts it receives, this platform can also be regarded as a repository of biomedical preprints, given that the initial text is published prior to undergoing this open peer-review process ${ }^{10}$. Another repository, PeerJ Preprints ${ }^{11}$, started operating by publishing its first preprints in April 2013, accounting to this day for several thousands of works in biomedicine.

In late 2013, as an answer to biomedical researchers' growing interest in AsXivi2, Cold Spring Harbor Laboratory launched a preprint server for biomedical areas named bioRXiv ${ }^{13}$. The bioRXiv platform gained notoriety when Nobel Prize winner, Carol Greider, from the Johns Hopkins University, uploaded a prepublication where she proposed a method for the regulation of telomere length ${ }^{14}$. Other much talked-about cases included prepublications by groups in the Cold Spring Harbor Laboratory, as the one of neuroscientist Steve Shea, whose manuscript had remained on peer-review for more than one and a half year by then ${ }^{15}$.

Once a work is submitted to bioRXiv, and before it is published in the internet, a basic filtering process for plagiarized, offensive, non-scientific or pseudoscientific content, but that doesn't include editing or 
typographic composition, is completed in a matter of hours. In addition, the repository policies establish that a work can be submitted prior or during a traditional peer-review process by a journal, but not after it has been published. The moment the text is published in the internet, it is assigned a digital object identifier (DOI), and revised versions if it can be submitted by the authors at any moment but, given that they are citable since their publication in the repository, the manuscripts cannot be withdrawn ${ }^{13}$. The entry corresponding to each preprint in bioRXiv includes the abstract text, hyperlinks to download the current version and the preprint previous versions in Pdf format, as well as an interface that enables citing it, sharing it in multiple social networks or via e-mail, as well as leaving comments after session is opened in Discus, Facebook, Twitter or Google. Furthermore, direct contact with authors via e-mail is promoted. In addition, preprints contain a prominent legend stating that the works have not been approved in a peer-review process and the repository warns that the articles it contains have not yet been accepted or endorsed whatsoever by the scientific community. Web addresses of the preprint repositories that are referred to in the text are presented in table 1.

\section{Advantages and disadvantages of the model}

Current publishing model enables that, relatively often, first notice of a discovery can be granted depending on the differences in peer-review promptness in different journals in the case of manuscripts reporting the same discovery, of comparable quality and complexity and submitted within a short time interval. Therefore, it has been proposed that the use of preprints would contribute to a fairer recognition in biomedical discoveries if simultaneously or subsequently accompanied by a rigorous peer-review ${ }^{16}$. However, this preprints use can be a double-edged sword. On one hand, it allows establishing the day of release in the repository as the point in time when the discovery is announced to the scientific community. In traditional journals, the date of manuscript first reception is usually stated; however it is only made public if and when the article is accepted. On the other hand, in areas of biomedicine that are highly competitive, a potential disadvantage of making the discovery public in a preprint would consist in alerting competitor groups with regard to the progress of a particular research and thereby accelerate their decision to submit their work
Table 1. Mentioned preprint repositories and their web addresses

\begin{tabular}{|c|c|c|c|}
\hline Repository & Web address & $\infty$ & \\
\hline ArXiv & http://arxiv.org/ & 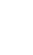 & \\
\hline bioR $\chi$ iv & http://biorxiv.org/ & 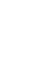 & \\
\hline F1000Research & \multicolumn{3}{|c|}{ http://f1000research.com/E } \\
\hline Nature Proceedings & \multicolumn{3}{|c|}{ http://precedings.nature.com/ } \\
\hline Netprints & \multicolumn{3}{|c|}{ http://staticweb.bmj.com/clinmed } \\
\hline PeerJ Preprints & \multicolumn{3}{|c|}{ http://peerj.com/preprints/ } \\
\hline
\end{tabular}

to a traditional pair-review. Inasmuch as preprint culture spreads, as it already happens in many exact sciences, reviewers might be more willing to verify the originality of an idea or discovery using both publications in journals and preprint repositories. Correspondingly, claiming first notice of a finding that has been previously reported in a preprint by another group or author without citing it, could become less socially acceptable.

In contrast with most biomedical journals, in the case of bioRXiv and other previous preprint repositories, publication of a work is free for authors. In addition, access, download and reading of these are free of cost for end-users. This enables the reported advances to be much more visible, citable and immediately usable by other investigators, potentially contributing to quicker advance of the field as a whole,

The assessment undergone by works submitted to repositories before they are made available to readers, for example, in bioRXiv, does not replace the pair-review process, and the quality of the works therein contained is therefore more variable in comparison with peer-reviewed journals. This implies that the responsibility to assess the validity, originality and importance of each work is transferred from reviewers to readers. This acquires special relevance for the use of preprints to measure researchers' performance, since verifying officers would be required to apply more analytical criteria to differentiate preprints of different degrees of quality and scientific rigor.

As described for the case of bioRXiv, biomedical preprint repositories' web interfaces facilitate for site users to comment about each work. This has the enormous advantage that anyone can contribute with criticism and suggestions to the authors, which would enable for a manuscript to be improved prior to submission to formal peer-review, a process where the content of each work is assessed by very few people. 
Currently, certain scientific journals impose limits to the number of words, figures and references to the articles they are submitted to, while others do not admit supplementary information inclusion. Since in bioRXiv these restrictions are not established, preprints might become valuable sources of detailed methodological descriptions, which would increase the possibilities for experiments to be able to be successfully reproduced.

The use of preprints can also contribute to decrease publication bias, in the sense that traditional journals are more likely to contain positive results. Under this premise, investigators may be more inclined to publish their negative results, a valuable source of information, in preprint repositories.

One difficulty for the practice of releasing preprints to be more extended is that, at this moment, some biomedical journals as, for example, those belonging to the Cell Publishing Group, do not accept articles that have been previously published in platforms such as bioRXiv. An online database, lodged in Wikipedia, keeps an updated registry about publishing houses policies with regard to acceptance of articles that have been the subject of preprint (https://en.wikipedia.org/ wiki/List_of_academic_journals_by_preprint_ policy).

\section{The ASAPbio Meeting}

Owing to the prominence preprints have been gaining in biomedicine, in part thanks to bioRXiv diffusion, around 70 renowned investigators, representatives of the industry, scientific financing agencies, as well as publishing houses representatives, all of them interested on this emergent system, met on mid-February 2016 during the Accelerating Science and Publication in biology (ASAPbio) meeting ${ }^{6}$ in order to discuss how the different types of stakeholders can favor rational and productive use of repositories and the texts therein stored. The academicians' analysis of conclusions highlights that, in order to promote preprints' production by biomedical investigators, it is necessary for systematic forms to exist, such as search engines, to associate an article published in a journal with the preprint(s) that preceded it. For financing agencies representatives, preprints can be valuable in order to have a more updated insight on a researcher's productivity, and a more opportune view on the reactions of the scientific community to his or her contributions. Publishing houses representatives concluded that preprints should play different, but complementary roles in the diffusion of scientific advances. They also specified that comments to a preprint might serve as a guidance to facilitate peer-reviewers' work, and that including mechanisms for preprint direct submission to traditional journals in the repositories would be advisable. In private and optional voting during the meeting, the vast majority chose favoring opinions that preprints can be used for first notice of a finding to be established, and that they also can be used as evidence in researchers' productivity assessments.

\section{Conclusions and final considerations}

Submitting a manuscript to a preprint server can be highly useful to improve its quality, thanks to the comments it receives, prior to submitting it to a journal for formal peer-review. In addition, preprints can be used to establish first notice of a discovery if and when, in the investigator's opinion, conclusions reached in the work are justified by the methodology and the results therein presented. Both aspects should be cautiously considered in highly competitive lines of research, where the risk of losing priority by small margins is high.

Inasmuch as preprints become established as an acceptable and visible means for biomedical scientific knowledge to be diffused, this instrument will be increasingly useful in order for these publications to become a criterion to grant professorships, stimuli and financing to researchers, a change that, however, should be accompanied by the acquisition of a critical focus by the verifying officers involved, in order to discriminate scientifically robust preprints from those that aren't.

Although the premise that, in biomedicine, current publication system in journals is broken beyond repair $^{17}$ is with no doubt catastrophist, it is undeniable that this process has been evolving much faster in recent years thanks to its conjunction with information technology and telecommunications immense advances. The use of preprints is but one of the innovations thereby fostered, and thanks to other types of platforms, such as repositories of data for open science and post-publishing peer-review platforms, among many others, it is possible that in the future we will observe the development of an entire ecosystem for the propagation of information generated by biomedical research. As a physicist, neuroscientist and co-discoverer of the DNA structure Francis Crick used to say, "In nature, hybrid species are usually sterile, but in science, the opposite is frequently true"18. 


\section{References}

1. Spier R. The history of the peer-review process. TRENDS Biotechnol. 2002:20:357-8

2. Goldbeck-Wood S. Evidence on peer review - scientific quality control or smokescreen? BMJ. 1999;318:44-5.

3. Powell K. Does it take too long to publish research? Nature. 2016:530:148-51.

4. Vale RD. Accelerating scientific publication in biology. Proc Natl Acad Sci U S A. 2015;112:13439-46.

5. Fang FC, Steen RG, Casadevall A. Misconduct accounts for the majority of retracted scientific publications. Proc Natl Acad Sci. 2012;109:17028-33.

6. Berg JM. Preprints for the Life Sciences. Science. 2016;352:899-901.

7. Homan JM. E-biomed. Bull Med Libr Assoc. 1999;87:485-6.

8. Delamothe T, Smith R, Keller MA. Netprints: the next phase in the evolution of biomedical publishing. BMJ. 1999;319:1515-6.

9. Free market science. Nat Cell Biol. 2007;9:721.

10. Ford E. Open peer review at four STEM journals: an observational overview. Version 2. F1000Res. 2015;4:6.
11. Callaway E. Biomedical journal and publisher hope to bring preprints to life. Nat Med. 2013;19:512

12. Callaway E. Geneticists eye the potential of arXiv. Nature. 2012;488:19.

13. Callaway E. Preprints come to life. Nature. 2013;503:180.

14. Greider CW. Regulating telomere length from the inside out: The replication fork model. Genes Dev. 2016;30(13):1483-91.

15. Krishnan K, Lau B, Ewall G, et al. MECP2 regulates cortical plasticity underlying a learned behavior in adult female mice. Nat Commun. 2017;8:14077.

16. Vale RD, Hyman AA. Priority of discovery in the life sciences. Elife. 2016;5.

17. Chalmers I, Glasziou P. Should there be greater use of preprint servers for publishing reports of biomedical science? F1000Research. 2016;5:272.

18. Crick F. What mad pursuit: a personal view of scientific discovery. Basic Books; 2008. 